\title{
Detection of obstructive sleep apnoea by an electronic nose
}

\author{
Timm Greulich¹, Akira Hattesohl', Antje Grabisch'1, Janine Koepke1, \\ Severin Schmid ${ }^{1}$, Sarah Noeske', Christoph Nell ${ }^{1}$, Marion Wencker ${ }^{2}$, \\ Rudolf A. Jörres ${ }^{3}$, Claus Franz Vogelmeier ${ }^{1}$, Ulrich Köhler ${ }^{1}$ and \\ Andreas Rembert Koczulla ${ }^{1}$
}

\author{
Affiliations: \\ ${ }^{1}$ Respiratory Medicine, University of Marburg, University Hospital of Gießen and Marburg, Marburg, \\ ${ }^{2}$ Dept of Pneumology, University Hospital Essen, Ruhrlandklinik, Essen, and \\ ${ }^{3}$ Institute and Outpatient Clinic for Occupational, Social and Environmental Medicine, Ludwig-Maximilians- \\ Universität, Munich, Germany.

\section{Correspondence:} \\ T. Greulich, Respiratory Medicine, University Hospital of Gießen and Marburg, 35043 Marburg, Germany. \\ E-mail: greulichamed.uni-marburg.de
}

ABSTRACT Diagnosis of obstructive sleep apnoea syndrome (OSAS) is technically demanding, costintensive and time-consuming. The measurement of volatile organic compounds by an electronic nose is an innovative method that determines distinct molecular patterns of exhaled breath in different patient groups. We addressed the following questions: What is the diagnostic accuracy of an electronic nose in the detection of OSAS and the ability to detect effects of standard therapy in patients with OSAS? Are these results related to changes in distinct markers of airway inflammation and extracellular remodelling?

We included 40 OSAS patients and 20 healthy controls. Exhaled breath of all participants was analysed using the Cyranose 320 electronic nose. Pharyngeal washings were performed to sample the upper airway compartment. For statistical analysis linear discriminant analysis was employed.

We identified a linear discriminant function separating OSAS from control $(\mathrm{p}<0.0001)$. The corresponding area under the receiver-operating curve was 0.85 (95\% CI $0.75-0.96$; sensitivity 0.93 and specificity 0.7 ). In pharyngeal washing fluids of OSAS patients, we observed higher levels of $\alpha_{1}$-antitrypsin and markers of extracellular remodelling compared to controls.

The electronic nose can distinguish between OSAS patients and controls with high accuracy.

-

@ERSpublications

The electronic nose can distinguish between OSAS patients and controls with high accuracy http://ow.ly/13d8s

For editorial comments see page 1 .

A press release for this article can be found at www.erj.ersjournals.com/site/misc/presspack.xhtml

This article has supplementary material available from www.erj.ersjournals.com

Received: June 102012 | Accepted after revision: Oct 042012 | First published online: Oct 252012

Support statement: This study was supported by the German Research Foundation (DFG), SFB/TR22 A19 and by a grant of the Behring-Roentgen Foundation (56-0036).

Conflict of interest: Disclosures can be found alongside the online version of this article at www.erj.ersjournals.com

Copyright @ERS 2013 


\section{Introduction}

Obstructive sleep apnoea syndrome (OSAS) is a common disease [1] associated with an increased risk for cardiovascular disorders [2, 3]. The current gold standard to confirm OSAS is multichannel polysomnography (PSG) [4]. This is technically demanding, time-consuming, and labour- and costintensive with limited availability.

Different screening tools have been developed to reduce the number of patients requiring PSG but most of them lack sensitivity and/or specificity. Clinical parameters such as the Epworth Sleepiness Scale, neck circumference and a composite clinical score show a large overlap between healthy controls and OSAS patients [5-7].

Technical tools sensing a change of body position as a surrogate for respiratory movements [8], sound analysis [9] and complex computerised analyses of ECG recordings [10] have been evaluated with different success rates. A novel screening tool predicting OSAS with acceptable accuracy and without overnight measurements could improve the OSAS screening algorithm.

Several studies have revealed that OSAS is associated with increased oxidative stress [11], as well as systemic and local inflammation [12], as indicated by increased concentrations of pro-inflammatory cytokines and other markers in the exhaled breath condensate (EBC) and serum [11]. The serum level of $\alpha_{1}$-antitrypsin $\left(\alpha_{1}-\mathrm{AT}\right)$, an acute phase reactant playing a major role in the control of inflammation [13], also seems to be elevated [14]. Other compounds such as matrix metalloproteases (MMPs) and tissue inhibitor of matrix metalloproteases (TIMPs) might also be involved in the disease process as mediators of the ongoing airway remodelling [15].

Exhaled breath contains hundreds of volatile organic compounds (VOCs), as demonstrated by mass spectrometry [16], and its analysis can provide information about systemic or local inflammation. Instead of identifying single compounds, the assessment of exhaled breath can also be performed by devices enabling the recognition of patterns of VOCs [17]. Indeed, such devices can distinguish between a number of diseases via their VOC profiles [18-20]. A recent review article summarising potential medical applications has been published elsewhere [21]. To our knowledge, VOC profiles have not been assessed in patients with OSAS.

Our hypothesis was that a pattern-recognising electronic nose is capable of distinguishing between OSAS patients and healthy controls. In addition, we assumed that therapy with continuous positive airway pressure (CPAP) has a detectable effect on airway inflammation and consequently the VOC profile. To substantiate the data by direct measurement of biochemical compounds, we assessed whether markers of inflammation and airway remodelling differ between healthy controls and OSAS before and/or after CPAP treatment. For this purpose we selected $\mathrm{pH}$ and conductivity of EBC, and MMP-9, TIMP-1 and $\alpha_{1}$-AT in pharyngeal washing fluids.

\section{Methods}

\section{Subjects and study design}

20 healthy volunteers were recruited from the hospital staff and 40 OSAS patients from a sleep apnoea outpatient clinic before receiving CPAP therapy. Inclusion criteria for healthy controls were the ability and willingness to participate. Volunteers with any known chronic disease or any acute disease in the last 4 weeks before study entry or any medication taken on a regular basis were excluded. OSAS was defined as an apopnoea/hypopnoea index (AHI) $>5$ events $\cdot \mathrm{h}^{-1}$ in combination with clinical signs of obstructive sleep apnoea. We excluded patients with any other chronic and/or acute respiratory disease of the upper and/or lower airways (e.g. asthma and chronic obstructive pulmonary disease (COPD)). These diseases were assessed by a questionnaire (patient-reported). In any case of doubt, lung function testing was performed. The following comorbid conditions were documented systematically: coronary heart disease, diabetes and arterial hypertension. Disease-specific medical therapy was allowed and left unaltered in the course of the study. The study was approved by the local ethics committee (Marburg Ethics Committee AZ 59/06 Amendment 3; Marburg, Germany) and written informed consent was obtained from each subject.

All participants had sleep studies and answered a questionnaire regarding symptoms, smoking habits, health status, medication and medical history. In addition, the following examinations were performed: collection of pharyngeal washing fluid, collection of EBC, measurement of exhaled breath with the Cyranose 320 electronic nose (Smiths Detection Group Ltd, Watford, UK). The first 20 of the 40 OSAS patients were examined again after 3 months of CPAP therapy.

\section{Polysomnography/polygraphy}

Patients with suspected OSAS underwent an overnight PSG (Embla N7000; TNI Medical AG, Bad Ems, Germany). Electroencephalogram, electrooculogram and electromyogram were measured using established 
procedures. Furthermore, thoracic and abdominal respiratory excursions, breath sounds, nasal airflow, ECG and oxygen saturation were recorded. In healthy controls, home polygraphy (SOMNOcheck2 R? Weinmann, Hamburg, Germany) was used to exclude OSAS. An AHI $<5$ events $\cdot \mathrm{h}^{-1}$ was defined as the absence of OSAS.

\section{Pharyngeal washing fluid}

All participants had to have been fasting for at least $2 \mathrm{~h}$, including no chewing gum or any kind of candy, and no tobacco smoking. They washed out their mouth with water before rinsing the throat with $25 \mathrm{~mL}$ of water by gargling. The fluid was stored at $-80^{\circ} \mathrm{C}$ for ELISA-based analysis of TIMP-1 and MMP-9 (R\&D Systems, Minneapolis, MN, USA), which were conducted according to the manufacturer's suggested routine procedure. $\alpha_{1}$-AT was measured by ELISA as described previously [22]. The sensitivity of the ELISAs for TIMP-1, MMP-9 and $\alpha_{1}$-AT was $30 \mathrm{pg} \cdot \mathrm{mL}^{-1}, 30 \mathrm{pg} \cdot \mathrm{mL}^{-1}$ and $40 \mathrm{pg} \cdot \mathrm{mL}^{-1}$, respectively.

$E B C$

EBC was collected by tidal breathing over 15 min using the ECoScreen Turbo (VIASYS; CareFusion Germany $234 \mathrm{GmbH}$, Höchberg, Germany) as described [23].

\section{Electronic nose}

The exhaled breath was assessed with the Cyranose 320 electronic nose. The participants breathed medicinal air (Aer medicinalis Linde; Linde Gas Therapeutics GmbH, Unterschleißheim, Germany) and exhaled for $10 \mathrm{~s}$ at a flow rate of $100-200 \mathrm{~mL} \cdot \mathrm{s}^{-1}$ into a disposable collection bag, which then was assessed within $60 \mathrm{~s}$. This medicinal air was also used as reference air for the 60-s baseline, followed by a 60-s sample draw from the collection bag, and completed by a 60-s purging of the electronic nose. These measurements were performed in triplicate [20].

\section{Data analysis}

The three data sets obtained by the electronic nose were averaged by taking their arithmetic mean for each individual. Principal component analysis was performed on these data. The resulting transformed data were fed into a linear discriminant analysis. The linear discriminant analysis results were then used for further analyses, including nonparametric statistical significance tests. The Mahalanobis distance between the groups was determined and a leave-one-out cross-validation of the data was performed to calculate the cross-validation value as described previously [24]. Additionally, a receiver operating characteristic (ROC) curve using the linear discriminant as a discriminative variable was constructed to determine the area under the curve (AUC). The values for sensitivity and specificity were derived from linear discriminant analysis "self-prediction" [25], meaning that the complete data set was used to calculate the values. To support this analysis we additionally performed a split-half analysis using the first 20 patients as the training set and the second half as the test set (and vice versa). Sensitivity and specificity were reported at the specific cut-off level, where the sum of the sensitivity and the specificity (Youden score) was highest [26].

Prior to the statistical comparisons, the data were checked for normal distribution by the KolmogorovSmirnov test. For data not normally distributed, nonparametric tests were used (Mann-Whitney U-test for unpaired and Wilcoxon matched-pairs signed-rank test for paired data), otherwise a t-test (unpaired or paired). To determine the correlation between the linear discriminant analysis and parameters of inflammation, the Spearman's rank correlation coefficient was calculated. Data are presented as mean \pm SD unless stated otherwise. The software GraphPad Prism 5.00 (GraphPad Inc., San Diego, CA, USA) and SPSS Version 20 (SPSS Inc., Chicago, IL, USA) was used for all statistical analyses.

\section{Results}

Patients versus controls

Baseline characteristics of the 40 OSAS patients and 20 healthy volunteers are shown in table 1.

The linear discriminant analysis scores of OSAS patients and healthy controls differed statistically significantly from each other $(\mathrm{p}<0.0001$; Mann-Whitney U-test) (fig. 1a). The Mahalanobis distance between the two groups was 1.88 and the cross-validation value $79.5 \%$. When using a split-half analysis, $80 \%$ of the "second half" was correctly predicted to have OSAS. Conversely, when predicting the "first half" after having used the second half as training set, $85 \%$ were correctly predicted to suffer from OSAS. The corresponding area under the ROC curve was 0.85 (95\% CI 0.745-0.960) (fig. 1b), indicating a sensitivity of 0.93 and a specificity of 0.70 . Furthermore, the linear discriminant analysis was significantly correlated with the AHI (Spearman's $\mathrm{r}=0.58, \mathrm{p}<0.001$ ), indicating a "dose-response" relationship. There was no significant correlation between the linear discriminant analysis and the other measured markers $(\mathrm{EBC} \mathrm{pH}$, EBC conductivity and inflammatory markers in pharyngeal washings). 
TABLE 1 Baseline characteristics of obstructive sleep apnoea syndrome (OSAS) patients and healthy controls

\begin{tabular}{|c|c|c|c|}
\hline & OSAS patients & Healthy controls & p-value \\
\hline Female/male $n$ & $3 / 37$ & $8 / 12$ & $<0.001^{\#}$ \\
\hline Age years & $55 \pm 10$ & $40 \pm 8$ & $<0.001^{\oplus}$ \\
\hline Height $\mathrm{cm}$ & $175 \pm 7$ & $175 \pm 8$ & 0.888 \\
\hline Weight kg & $99 \pm 15$ & $78 \pm 14$ & $<0.001^{\oplus}$ \\
\hline $\mathrm{BMI} \mathbf{k g} \cdot \mathrm{m}^{-2}$ & $32.00 \pm 4.5$ & $25.4 \pm 4.1$ & $<0.001^{\oplus}$ \\
\hline AHI events $\cdot h^{-1}$ & $33.65 \pm 22.00$ & $2.7 \pm 1.7$ & $<0.001^{\oplus}$ \\
\hline Al events $\cdot h^{-1}$ & $11.9 \pm 16.7$ & $0.6 \pm 1.1$ & $<0.001^{+}$ \\
\hline HI events $\cdot h^{-1}$ & $22.6 \pm 19.5$ & $2.1 \pm 1.5$ & $<0.001^{\top}$ \\
\hline $\mathrm{SO}_{2} \%$ & $92.8 \pm 2.7$ & $95.5 \pm 1.1$ & $0.01^{\top}$ \\
\hline
\end{tabular}

Data are presented as mean \pm SD, unless otherwise stated. BMI: body mass index, AHI: apnoea/hypopnoea index; Al: apnoea index; $\mathrm{HI}$ : hypopnoea index; $\mathrm{SO}_{2}$ : oxygen saturation. " : Fisher's exact test; ": t-test; ${ }^{+}$: MannWhitney U-test.

EBC $\mathrm{pH}$ values $(8.16 \pm 0.47$ in OSAS versus $8.09 \pm 0.40$ in healthy controls; $\mathrm{p}=0.27$; $\mathrm{t}$-test $)$ and $\mathrm{EBC}$ conductivity $\left(175.5 \pm 292.9 \mu \mathrm{S} \cdot \mathrm{cm}^{-1}\right.$ in OSAS versus $93 \pm 33.36 \mu \mathrm{S} \cdot \mathrm{cm}^{-1}$ in healthy controls; $\mathrm{p}=0.17$; $\mathrm{t}$-test $)$ of both groups did not differ significantly (fig. 2).

The $\alpha_{1}$-AT concentrations in pharyngeal washing fluids were significantly higher in OSAS patients compared with healthy controls $\left(60.6 \pm 52.0 \mu \mathrm{g} \cdot \mathrm{mL}^{-1}\right.$ versus $25.3 \pm 21.7 \mu \mathrm{g} \cdot \mathrm{mL}^{-1} ; \mathrm{p}=0.007$; t-test) (fig. $\left.3 \mathrm{a}\right)$. In contrast,
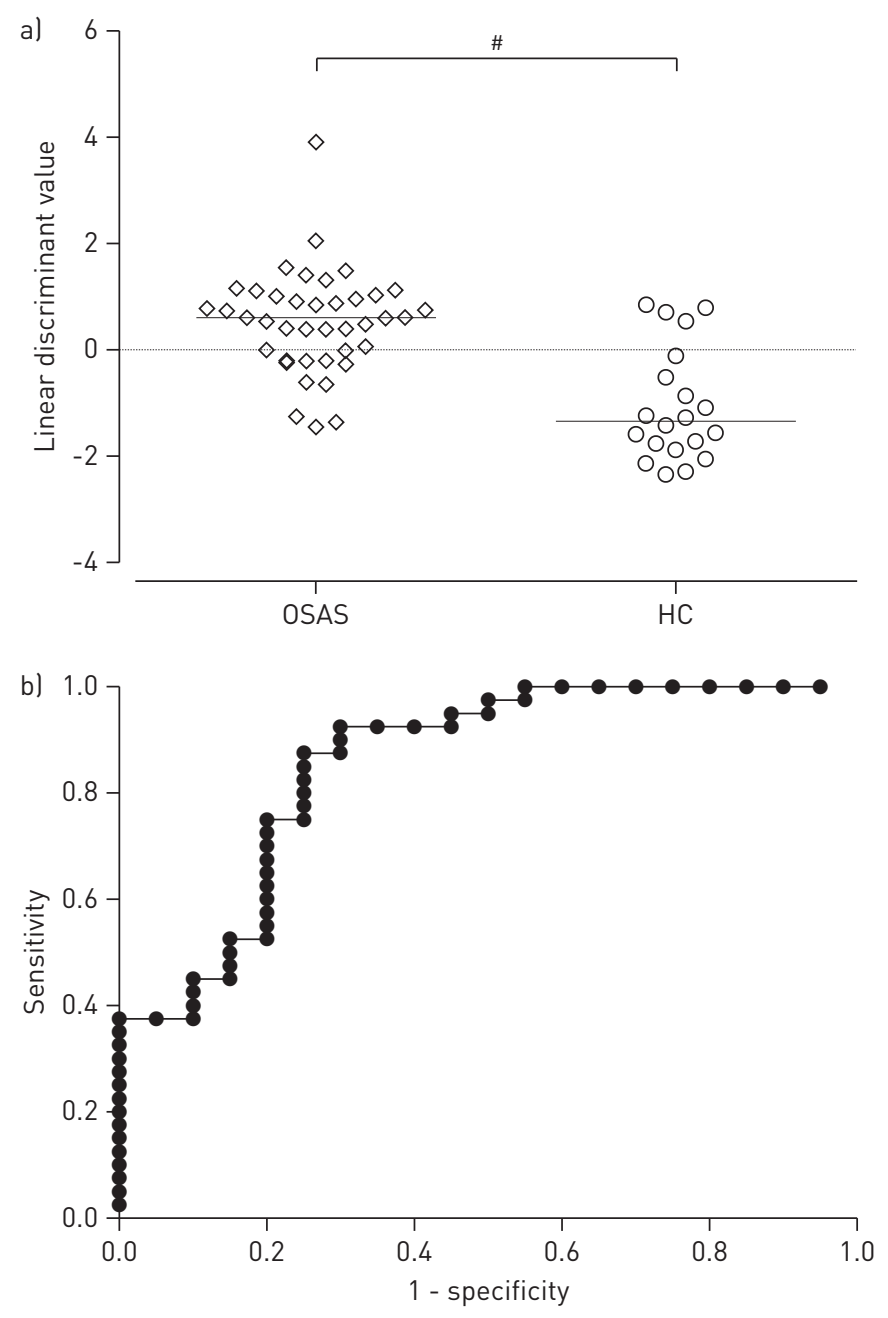

FIGURE 1 a) Linear discriminant analysis of sleep apnoea patients and healthy controls differ statistically significantly. b) The area under the receiver operating characteristic curve equals 0.85 , resulting in a sensitivity of 0.93 and a specificity of 0.70 . OSAS: obstructive sleep apnoea syndrome; HC: healthy control. \#: $p<0.0001$, MannWhitney U-test. 
FIGURE 2 The exhaled breath condensate values of a) $\mathrm{pH}$ and b) conductivity do not differ statistically significantly $(\mathrm{p}=0.27$ and $\mathrm{p}=0.17$, respectively, $\mathrm{t}-$ test). OSAS: obstructive sleep apnoea syndrome; HC: healthy controls.
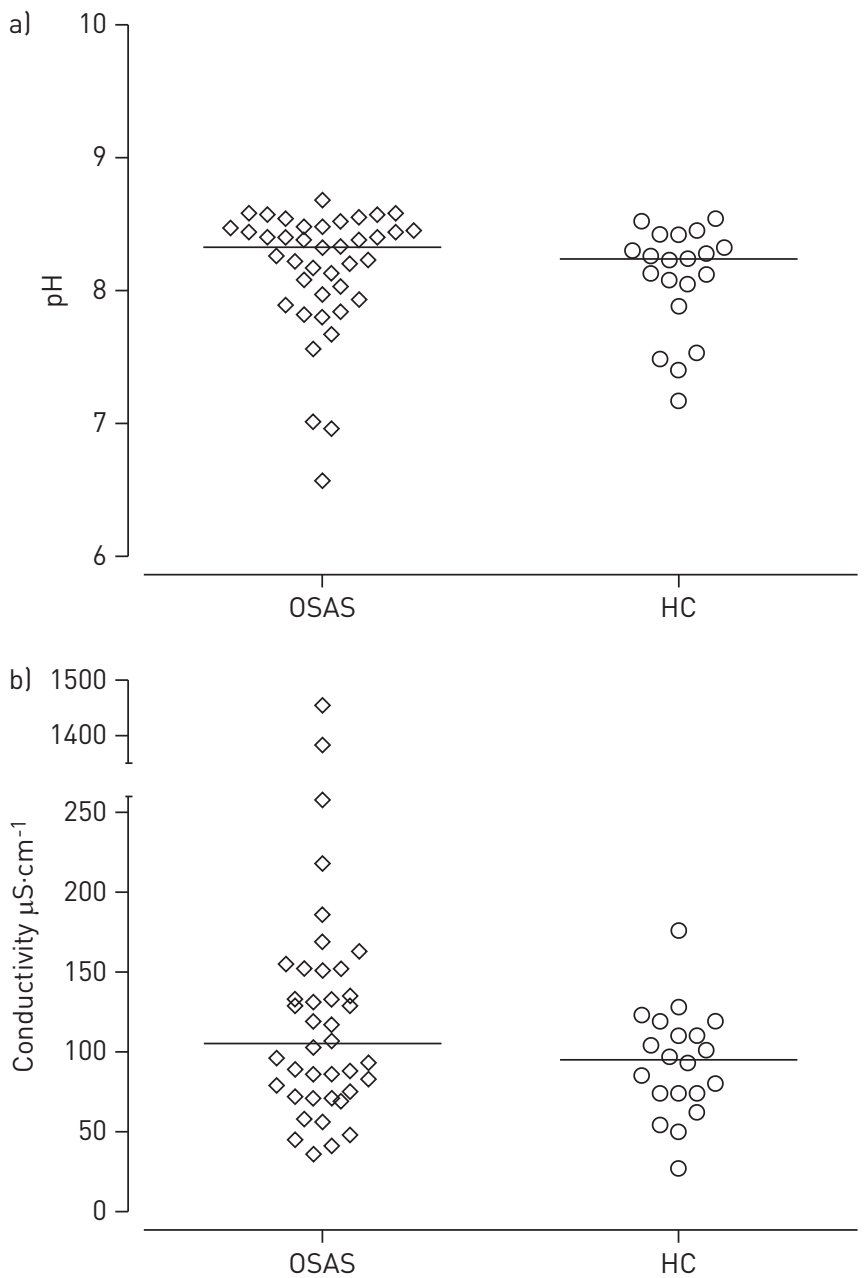

the difference in concentrations for MMP-9 (OSAS $5077.3 \pm 9104.5 \mathrm{pg} \cdot \mathrm{mL}^{-1}$ versus healthy controls 1008.6 $\pm 872.9 \mathrm{pg} \cdot \mathrm{mL}^{-1} ; \mathrm{p}=0.06$; t-test) and TIMP-1 (OSAS 7918.9 $\pm 7075.7 \mathrm{pg} \cdot \mathrm{mL}^{-1}$ versus healthy controls $6961.6 \pm 11412 \mathrm{pg} \cdot \mathrm{mL}^{-1} ; \mathrm{p}=0.16$; Mann-Whitney U-test) did not reach statistical significance. However, the MMP-9/TIMP-1 ratio showed a significant difference between groups (OSAS $0.69 \pm 1.11$ versus healthy controls $0.24 \pm 0.25 ; \mathrm{p}=0.02$; Mann-Whitney U-test) (fig. 3b). Compared with the VOC analysis, any other marker (EBC $\mathrm{pH}, \mathrm{EBC}$ conductivity and any marker in pharyngeal washing fluid) was inferior in predicting OSAS (AUC of the ROC curves ranged from 0.59 to 0.71 ; data not shown). However, the combination of inflammatory markers in pharyngeal washings and $\mathrm{EBC} \mathrm{pH} /$ conductivity with the linear discriminant analysis increased the diagnostic accuracy to $100 \%$ (AUC of the ROC 1, 95\% CI 0.94-1.00).

\section{Patients before and after 3 months of CPAP therapy}

The characteristics of the first 20 OSAS patients measured before and after initiation of CPAP therapy are listed in table 2.

The linear discriminant analysis values of before and after initiation of standard CPAP therapy differed significantly ( $\mathrm{p}=0.0003$; Wilcoxon test) (fig. $4 \mathrm{a}$ ). The Mahalanobis distance between the two groups was 1.83 , the cross-validation value $63.1 \%$ and the corresponding area under the ROC curve $0.82(95 \% \mathrm{CI}$ $0.6825-0.9475$ ) (fig. 4b), with a sensitivity of 0.80 and a specificity of 0.65 .

EBC $\mathrm{pH}$ values of both visits were similar (pre- $8.08 \pm 0.52$ versus post-initiation $8.05 \pm 0.85 ; \mathrm{p}=0.63$, Wilcoxon test) (fig. 5a); however, conductivity differed (pre- $186.7 \pm 303.6 \mu \mathrm{S} \cdot \mathrm{cm}^{-1}$ versus post-initiation $97.9 \pm 59.35 \mu \mathrm{S} \cdot \mathrm{cm}^{-1} ; \mathrm{p}<0.05$, Wilcoxon test) (fig. 5b).

Moreover, the $\alpha_{1}$-AT concentration decreased significantly after CPAP treatment (pre- $66.3 \pm 48.4 \mu \mathrm{g} \cdot \mathrm{mL}^{-1}$ versus post-initiation $42.8 \pm 36.3 \mu \mathrm{g} \cdot \mathrm{mL}^{-1}$; $\mathrm{p}=0.017$, paired t-test) (fig. 6a). For MMP-9 (pre- $7785.6 \pm 10500 \mathrm{pg} \cdot \mathrm{mL}^{-1}$ 

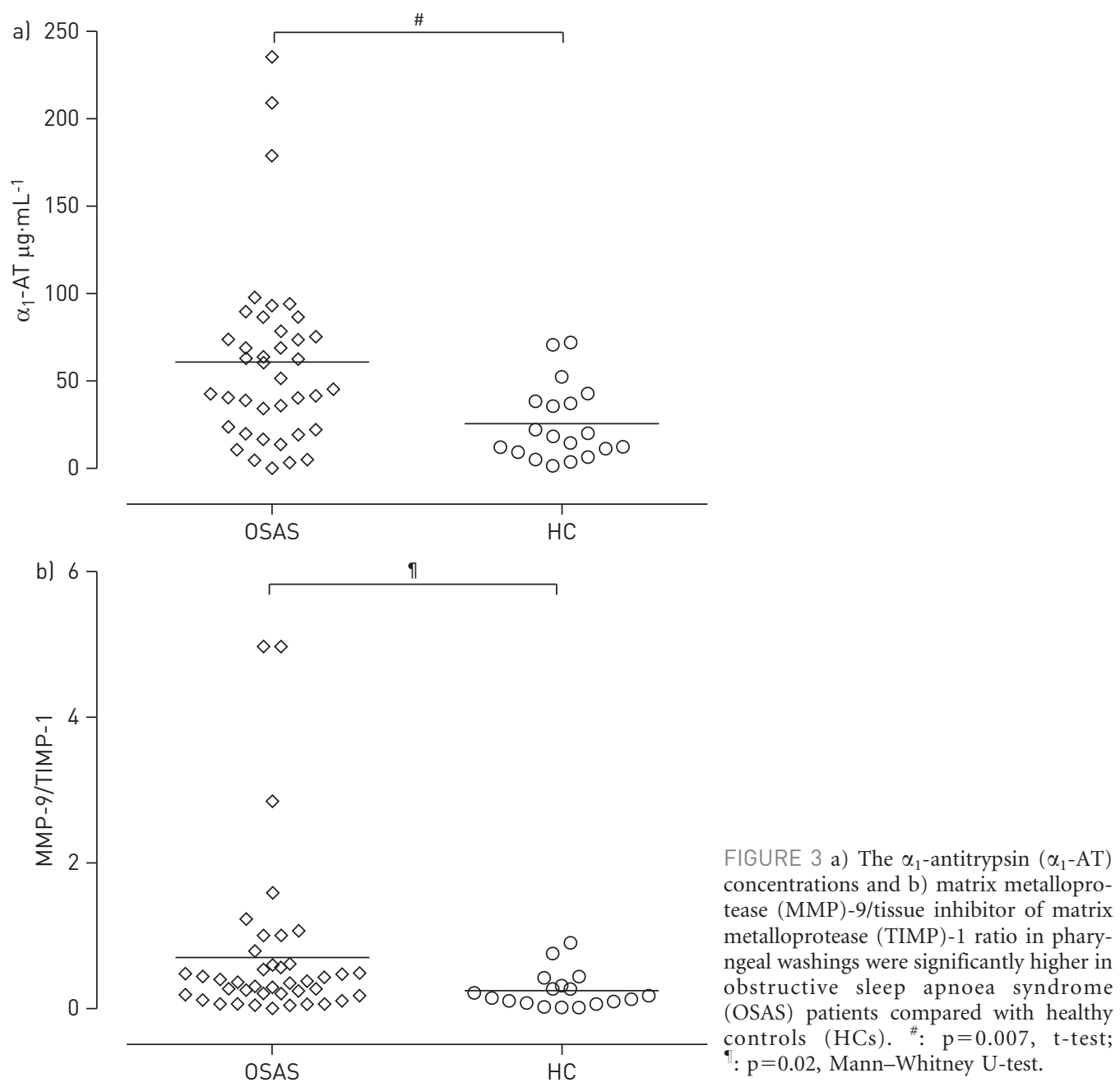

versus post-initiation $9185.8 \pm 11003 \mathrm{pg} \cdot \mathrm{mL}^{-1} ; \mathrm{p}=0.421$, Wilcoxon test) and TIMP-1 (pre$8344.7 \pm 7201.1 \mathrm{pg} \cdot \mathrm{mL}^{-1}$ versus post-initiation $13201 \pm 13151 \mathrm{pg} \cdot \mathrm{mL}^{-1} ; \mathrm{p}=0.314$, Wilcoxon test) there was no significant difference. The same applied to the MMP-9/TIMP-1 ratio (pre- $0.87 \pm 1.2$ versus post-initiation $0.98 \pm 0.97 ; \mathrm{p}=0.37$, Wilcoxon test) (fig. 6b).

TABLE 2 Characteristics of the subgroup of obstructive sleep apnoea syndrome (OSAS) patients with measurements at baseline and after 3 months of continuous positive airway pressure therapy

OSAS patients

\begin{tabular}{|c|c|c|}
\hline & Pre-initiation & After 3 months \\
\hline Female/male n & & \\
\hline Age years & & \\
\hline Height $\mathrm{cm}$ & & \\
\hline Weight kg & & \\
\hline $\mathrm{BMI} \mathrm{kg} \cdot \mathrm{m}^{-2}$ & & \\
\hline AHI events $\cdot h^{-1}$ & $32.0 \pm 22.8$ & $2.9 \pm 3.4$ \\
\hline Al events $\cdot h^{-1}$ & $9.4 \pm 17.4$ & $1.3 \pm 2.8$ \\
\hline HI events $\cdot h^{1}$ & $22.6 \pm 21.7$ & $1.7 \pm 2.1$ \\
\hline $\mathrm{SO}_{2} \%$ & $92.5 \pm 2.5$ & $94.4 \pm 1.5$ \\
\hline
\end{tabular}

Data are presented as mean \pm SD. BMI: body mass index; AHI: apnoea/hypopnoea index; Al: apnoea index; HI: hypopnoea index; $\mathrm{SO}_{2}$ : oxygen saturation. 
FIGURE 4 a) The (paired) values of the linear discriminant analysis of sleep apnoea patients before (pre-initiation) and 3 months after (post-initiation) initiation of continuous posititve airway pressure therapy differ statistical significantly. b) The area under the receiver operating characteristic curve equals 0.82 , resulting in a sensitivity of 0.80 and a specificity of 0.65 . ${ }^{* * *}$ : $\mathrm{p}<0.001$, Wilcoxon signed-rank

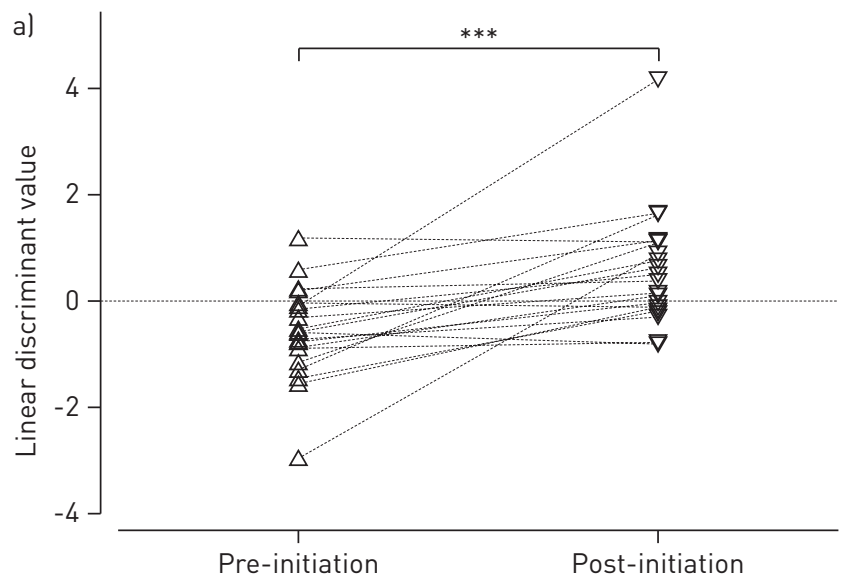
test.

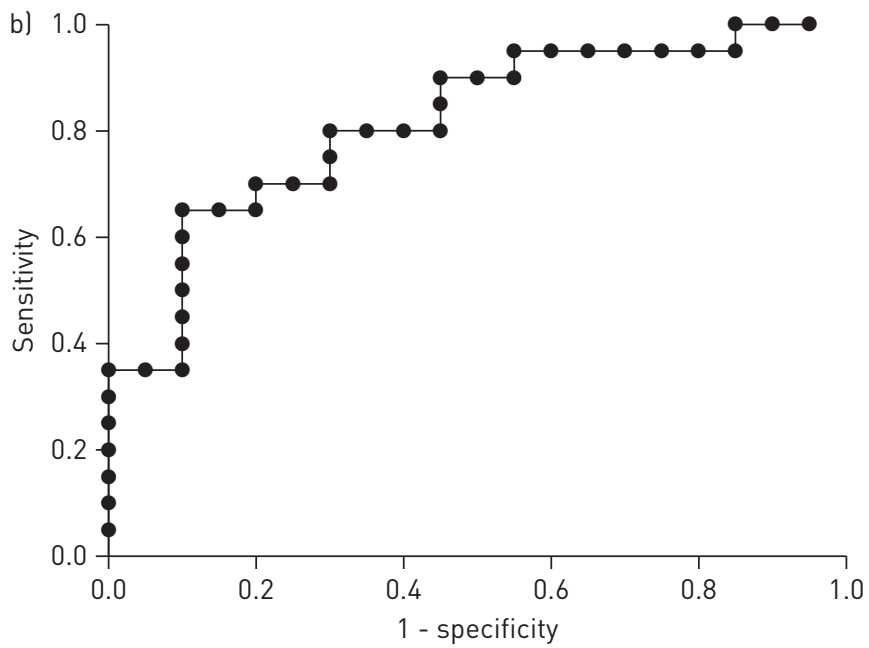

\section{Discussion}

This study shows that the Cyranose 320 electronic nose can distinguish the pattern of VOCs present in the exhaled breath of patients with OSAS from that of healthy subjects. A significant correlation between the linear discriminant and the AHI could be observed. Furthermore, the electronic nose could discriminate the state before and after treatment with CPAP. This indicates that specific VOC patterns in exhaled breath are associated with untreated OSAS.

Exhaled breath analysis for VOCs is a relatively novel option to obtain information about diseases. Gas chromatography and mass spectrometry have been used in the past [16]. The simpler, pattern-recognising electronic noses allow the rapid recognition of VOC mixtures in terms of "smellprints" [27], but not the identification of individual molecular components [17].

Electronic noses of various sophistications have been tested in a variety of respiratory diseases. In principle, the diagnosis of ear, nose and throat infections [28] or pneumonia [29] is possible. Moreover, patients with lung cancer [30], asthma [31], COPD [18] or $\alpha_{1}$-AT deficiency [20] could be recognised when compared with healthy controls or individuals with other respiratory diseases. In our study, we aimed to test the hypothesis that OSAS could be recognised by its VOC profile and that the profile would change after CPAP treatment. To our knowledge, this is the first study in which the smellprints of OSAS patients have been compared with healthy subjects.

Our results might be of interest, as nearly all screening tools for OSAS require overnight measurements. Although the available devices are easy to handle, some of the recordings exhibit poor quality, thus limiting their diagnostic value. Furthermore, some patients are unwilling to sleep while "connected to an electronic device". Conversely, overnight polygraphy with a limited number of channels does not require expensive medical staff, simply two patient visits to the clinic. Thus a diagnosis from other sources, e.g. "within a breath", would be desirable. 

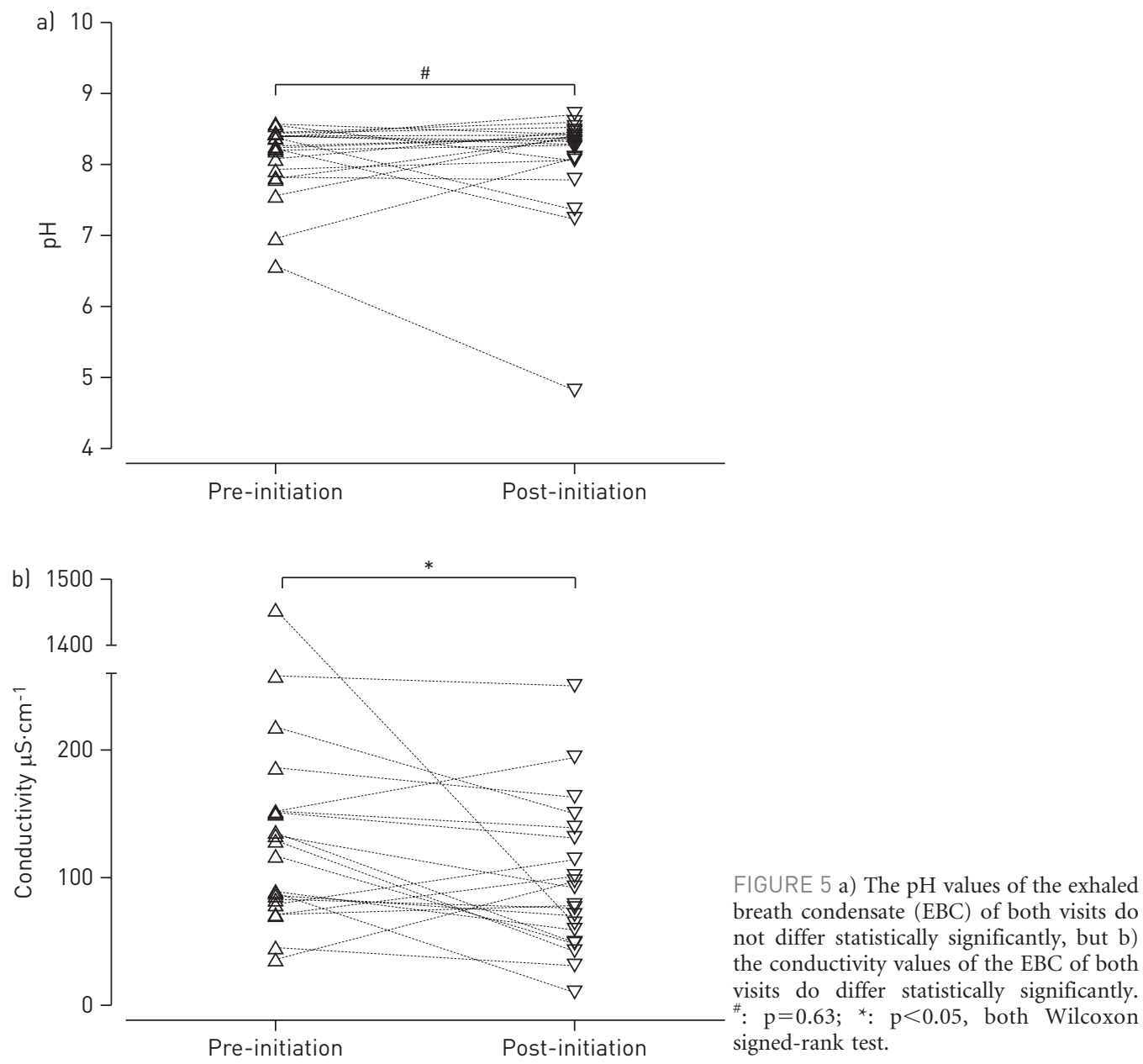

One might wish that single substances could be described to predict differences in the exhaled breath profile of different patient groups. While gas chromatography and/or mass spectrometry could achieve that, due to the tremendous costs and time-consuming data analysis, these methods would not be feasible in a clinical setting. The idea to describe (on a computational level) patterns without knowing the disease-specific substance is a promising new attempt with a clinically directed on-site approach.

It could be argued that an AUC of 0.85 is not sufficient for a diagnostic tool. However, diagnostic tests are used in specific clinical situations. The electronic nose could be useful on two occasions. First, to rule out the disease in a low prevalence population, e.g. in a general practitioner's office where the prevalence of OSAS is $\sim 2-4 \%$ [1]. A negative result would have a negative predictive value of $99.6 \%$ (95\% CI 90.3-100\%) and thus provide a high degree of certainty. Secondly, the device could be a decision aid with which to conduct overnight PSG. In a population with a high prevalence of OSAS (as high as 35\% in obese, snoring subjects), a positive result would have a positive predictive value of $62.4 \%$ (95\% CI $43.3-79.1 \%$ ) and would pave the way towards overnight PSG. Conversely, a negative result would have a negative predictive value of 94.5\% (95\% CI 78.9-99.6\%), thus drawing the attention to diagnoses other than OSAS. Furthermore, a value of 0.85 for an AUC is in the range of other diagnostic tests that are used in daily clinical practice, such as troponin for the diagnosis of myocardial infarction (AUC of 0.87 [32]).

To reveal whether there would be specific chemical or physicochemical alterations in OSAS patients, we also analysed EBC, focussing on easily accessible markers such as $\mathrm{pH}$ and electrical conductivity, which we have recently found to be robust and reproducible markers unaltered by respiratory manoeuvres [24]. EBC $\mathrm{pH}$ values were in the range of 8 , which is comparable with what has been reported before [33-35]. However, we did not find major differences in these measures, which might be too unspecific for the disease.

In contrast, $\alpha_{1}$-AT in pharyngeal washing fluid as a marker of inflammation showed elevated values in OSAS patients and these were reduced after only 3 months of CPAP treatment. This could reflect the response to the intermittent hypoxia and/or cyclic sheer forces that are alleviated by CPAP. Similar results 
FIGURE 6 a) The $\alpha_{1}$-antitrypsin $\left(\alpha_{1}\right.$ AT) concentration in pharyngeal washing fluids decreased significantly after 3 months of continuous positive airway pressure treatment. \#: $\mathrm{p}=0.017$, t-test for paired samples. b) Matrix metalloprotease (MMP)-9/tissue inhibitor of matrix metalloprotease (TIMP)-1 ratio did not change significantly in obstructive sleep apnoea syndrome patients before (pre-initiation) and after (post-initiation) treatment $(\mathrm{p}=0.37$, Wilcoxon signed-rank test).
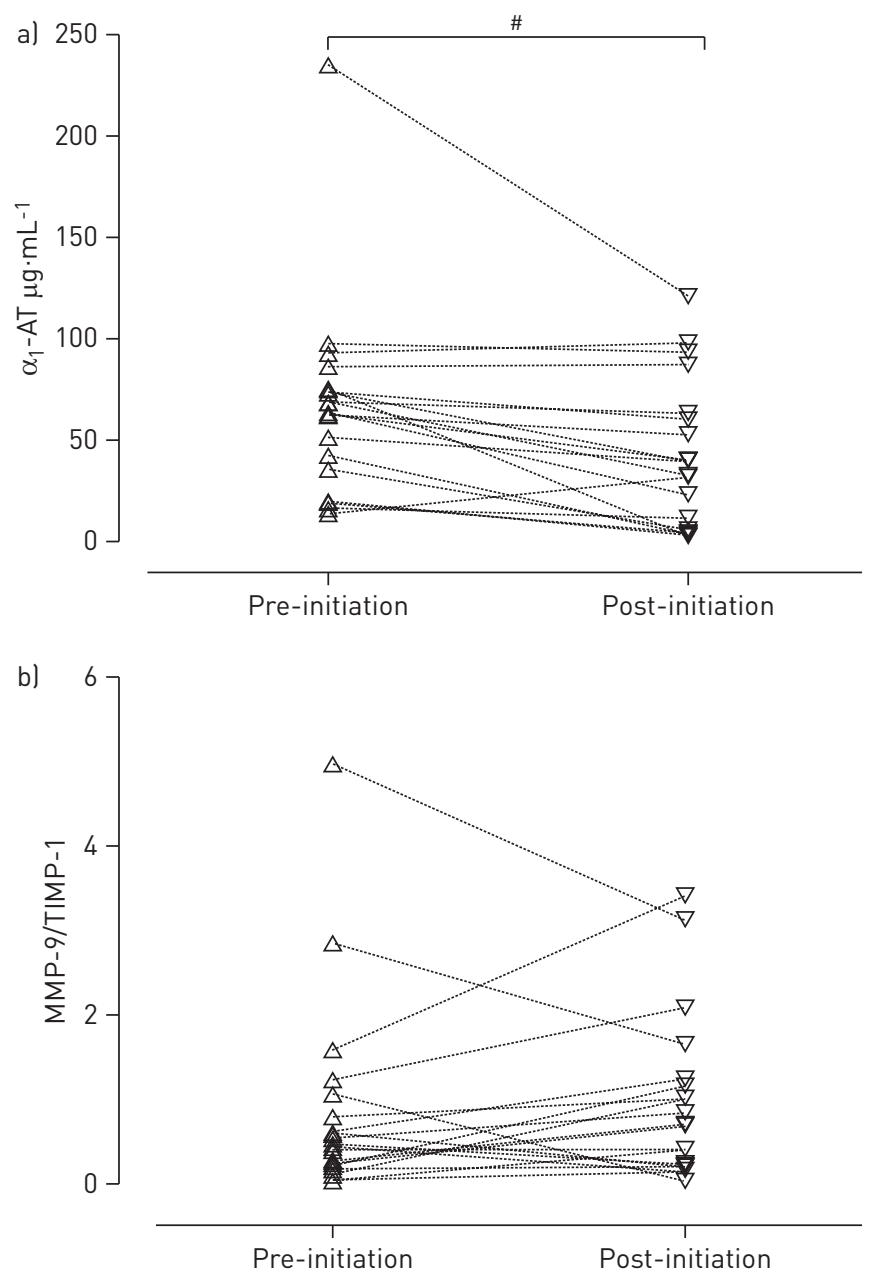

have been shown for local and systemic markers of inflammation and/or oxidative stress [36, 37]. We used MMP-9, TIMP-1 and their ratio MMP-9/TIMP-1 as further markers; the ratio showed elevated levels in OSAS. To our knowledge, this is the first time that the MMP-9/TIMP-1 ratio has been examined in a local compartment in OSAS. On a systemic level, increased MMP-9 concentration and activity have already been described in OSAS and these alterations could be reduced by CPAP therapy [38]. This leads us to believe that the increased ratio of MMP-9/TIMP-1 is an indicator of upper airway remodelling following chronic intermittent hypoxia and sheer forces.

This study has a number of limitations. The groups were not matched for age and body mass index (BMI), both of which are known to be potential confounders in exhaled breath analysis. To investigate whether age and BMI were confounders for the altered VOC mixture, we performed a logistic regression analysis with $\mathrm{AHI}$ as the dependent variable. The electronic nose-derived linear discriminant analysis was the best and solely significant predictor of AHI (OR 3.03, 95\% CI 1.41-6.51) compared with BMI (OR 1.17, 95\% CI $0.99-1.37$ ) and age (OR 1.05, 95\% CI 0.98-1.12). This was also reflected in a significant correlation between AHI and linear discriminant analysis (Spearman's $r=0.58, p<0.001$ ). Moreover, the significant change of the VOC profile before versus after CPAP treatment suggests that the differences between groups in the VOC profile were mainly due to the presence of OSAS. This represents a major advantage compared with previous publications where the VOC profile was assessed only once and significant correlations to other biological markers were not demonstrated.

Because of the high prevalence of comorbid conditions in OSAS patients, it could be argued that the altered VOC mixture was mainly due to comorbidities and/or associated medication. However, looking at the linear discriminant analysis graph (fig. 1a) and marking patients with coronary heart disease $(n=3)$, diabetes $(n=5)$ and arterial hypertension $(n=27)$, there was no trend in the distribution of patients with comorbidities (see online supplementary material). 
The data on inflammatory markers in pharyngeal washing are limited by missing standardised procedures to assess the absolute values of specific markers. HerR et al. [39] described defensin measurements in smokers, and pharyngeal washings have been used for the detection of potential respiratory pathogens [40]. Irrespective of this, the data for $\alpha_{1}$-AT strengthen the assumption that $\alpha_{1}$-AT is upregulated in untreated OSAS and pharyngeal washings are helpful to investigate this further.

Additionally, a sham CPAP and follow-up data would have strengthened our study and would have added data about the repeatability of the breathprint over time. However, the focus of this proof-of-concept study was on the diagnostic approach, with the goal of demonstrating the general possibility of the diagnostic potential of exhaled breath analysis regarding OSAS. We used the limited before/after comparison of exhaled breath to show that standard therapy changes the breathprint and to underline the assumption that the observed exhaled breath profile differences of OSAS versus healthy controls were mainly due to the presence/absence of obstructive episodes.

Most importantly, the results have to be validated in a separate cohort, possibly in an independent centre, in line with the Standards for the Reporting of Diagnostic accuracy studies (STARD) statement for the validation of diagnostic tests [41].

We conclude that the Cyranose 320 electronic nose is capable of distinguishing the exhaled breath of OSAS patients and control subjects with high accuracy.

\section{Acknowledgements}

Silke Mronga and Angelique Holland (University of Marburg, University Hospital of Gießen and Marburg, Marburg, Germany) replaced the first and senior authors in their clinical duties in 2010 and 2011. Otherwise the work would not have been possible.

\section{References}

Young T, Palta M, Dempsey J, et al. The occurrence of sleep-disordered breathing among middle-aged adults. $N$ Engl $J$ Med 1993; 328: 1230-1235.

2 Arzt M, Young T, Finn L, et al. Association of sleep-disordered breathing and the occurrence of stroke. Am J Respir Crit Care Med 2005; 172: 1447-1451.

3 Lattimore JD, Celermajer DS, Wilcox I. Obstructive sleep apnea and cardiovascular disease. J Am Coll Cardiol 2003; 41: 1429-1437.

4 Kushida CA, Littner MR, Morgenthaler T, et al. Practice parameters for the indications for polysomnography and related procedures: an update for 2005. Sleep 2005; 28: 499-521.

Bloch KE, Schoch OD, Zhang JN, et al. German version of the Epworth Sleepiness Scale. Respiration 1999; 66: 440-447. Davies RJ, Ali NJ, Stradling JR. Neck circumference and other clinical features in the diagnosis of the obstructive sleep apnoea syndrome. Thorax 1992; 47: 101-105.

$7 \quad$ Williams AJ, Yu G, Santiago S, et al. Screening for sleep apnea using pulse oximetry and a clinical score. Chest 1991; 100: 631-635.

8 Agatsuma T, Fujimoto K, Komatsu Y, et al. A novel device (SD-101) with high accuracy for screening sleep apnoeahypopnoea syndrome. Respirology 2009; 14: 1143-1150.

9 Karunajeewa AS, Abeyratne UR, Hukins C. Multi-feature snore sound analysis in obstructive sleep apnea-hypopnea syndrome. Physiol Meas 2011; 32: 83-97.

10 Corthout J, Van Huffel S, Mendez MO, et al. Automatic screening of obstructive sleep apnea from the ECG based on empirical mode decomposition and wavelet analysis. Conf Proc IEEE Eng Med Biol Soc 2008; 2008: 3608-3611.

11 Carpagnano GE, Spanevello A, Sabato R, et al. Systemic and airway inflammation in sleep apnea and obesity: the role of ICAM-1 and IL-8. Transl Res 2010; 155: 35-43.

12 Boyd JH, Petrof BJ, Hamid Q, et al. Upper airway muscle inflammation and denervation changes in obstructive sleep apnea. Am J Respir Crit Care Med 2004; 170: 541-546.

13 Janciauskiene SM, Bals R, Koczulla R, et al. The discovery of $\alpha_{1}$-antitrypsin and its role in health and disease. Respir Med 2011; 105: 1129-1139.

14 Sariman N, Levent E, Cubuk R, et al. Bronchial hyperreactivity and airway wall thickening in obstructive sleep apnea patients. Sleep Breath 2011; 15: 341-350.

15 Mattos W, Lim S, Russell R, et al. Matrix metalloproteinase-9 expression in asthma: effect of asthma severity, allergen challenge, and inhaled corticosteroids. Chest 2002; 122: 1543-1552.

16 Moser B, Bodrogi F, Eibl G, et al. Mass spectrometric profile of exhaled breath - field study by PTR-MS. Respir Physiol Neurobiol 2005; 145: 295-300.

17 Thaler ER, Hanson CW. Medical applications of electronic nose technology. Expert Rev Med Devices 2005; 2: 559-566.

18 Dragonieri S, Annema JT, Schot R, et al. An electronic nose in the discrimination of patients with non-small cell lung cancer and COPD. Lung Cancer 2009; 64: 166-170.

19 Fens N, Zwinderman AH, van der Schee MP, et al. Exhaled breath profiling enables discrimination of chronic obstructive pulmonary disease and asthma. Am J Respir Crit Care Med 2009; 180: 1076-1082.

20 Hattesohl AD, Jorres RA, Dressel H, et al. Discrimination between COPD patients with and without $\alpha_{1}$-antitrypsin deficiency using an electronic nose. Respirology 2011; 16: 1258-1264.

21 Wilson AD, Baietto M. Advances in electronic-nose technologies developed for biomedical applications. Sensors (Basel) 2011; 11: 1105-1176.

22 Koczulla AR, Noeske S, Herr C, et al. $\alpha_{1}$-Antitrypsin is elevated in exhaled breath condensate and serum in exacerbated COPD patients. Respir Med 2012; 106: 120-126. 

breath condensate in current smokers. Respiration 2010; 79: 61-67.

24 Huttmann EM, Greulich T, Hattesohl A, et al. Comparison of two devices and two breathing patterns for exhaled breath condensate sampling. PLoS One 2011; 6: e27467.

25 Altman DG, Bland JM. Diagnostic tests. 1: Sensitivity and specificity. BMJ 1994; 308: 1552.

26 Youden WJ. Index for rating diagnostic tests. Cancer 1950; 3: 32-35.

27 Lewis NS. Comparisons between mammalian and artificial olfaction based on arrays of carbon black-polymer composite vapor detectors. Acc Chem Res 2004; 37: 663-672.

28 Shykhon ME, Morgan DW, Dutta R, et al. Clinical evaluation of the electronic nose in the diagnosis of ear, nose and throat infection: a preliminary study. J Laryngol Otol 2004; 118: 706-709.

29 Hockstein NG, Thaler ER, Lin Y, et al. Correlation of pneumonia score with electronic nose signature: A prospective study. Ann Otol Rhinol Laryngol 2005; 114: 504-508.

30 Machado RF, Laskowski D, Deffenderfer O, et al. Detection of lung cancer by sensor array analyses of exhaled breath. Am J Respir Crit Care Med 2005; 171: 1286-1291.

31 Dragonieri S, Schot R, Mertens BJ, et al. An electronic nose in the discrimination of patients with asthma and controls. J Allergy Clin Immunol 2007; 120: 856-862.

32 Reiter M, Twerenbold R, Reichlin T, et al. Early diagnosis of acute myocardial infarction in patients with preexisting coronary artery disease using more sensitive cardiac troponin assays. Eur Heart J 2012; 33: 988 -997.

33 Profita M, La Grutta S, Carpagnano E, et al. Noninvasive methods for the detection of upper and lower airway inflammation in atopic children. J Allergy Clin Immunol 2006; 118: 1068-1074.

34 Koczulla R, von Degenfeld G, Kupatt C, et al. An angiogenic role for the human peptide antibiotic LL-37/hCAP-18. J Clin Invest 2003; 111: 1665-1672.

35 Koczulla R, Dragonieri S, Schot R, et al. Comparison of exhaled breath condensate pH using two commercially available devices in healthy controls, asthma and COPD patients. Respir Res 2009; 10: 78.

36 Carpagnano GE, Kharitonov SA, Resta O, et al. 8-Isoprostane, a marker of oxidative stress, is increased in exhaled breath condensate of patients with obstructive sleep apnea after night and is reduced by continuous positive airway pressure therapy. Chest 2003; 124: 1386-1392.

37 Lavie L, Lavie P. Molecular mechanisms of cardiovascular disease in OSAHS: the oxidative stress link. Eur Respir J 2009; 33: 1467-1484.

38 Tazaki T, Minoguchi K, Yokoe T, et al. Increased levels and activity of matrix metalloproteinase-9 in obstructive sleep apnea syndrome. Am J Respir Crit Care Med 2004; 170: 1354-1359.

39 Herr C, Beisswenger C, Hess C, et al. Suppression of pulmonary innate host defence in smokers. Thorax 2009; 64: $144-149$.

40 Lieberman D, Shleyfer E, Castel H, et al. Nasopharyngeal versus oropharyngeal sampling for isolation of potential respiratory pathogens in adults. J Clin Microbiol 2006; 44: 525-528.

41 Bossuyt PM, Reitsma JB. The STARD initiative. Lancet 2003; 361: 71 . 